\title{
$\mathbb{A}$ Economics Bulletin
}

Volume 36, Issue 2

\section{Giving away the store: How the zero price constraint results in fewer add-on features}

\author{
Ben O Smith \\ University of Nebraska at Omaha
}

\begin{abstract}
This paper discusses an issue impacting intellectual property products with nearly zero marginal cost: the zero price constraint. As established in the literature, aftermarkets result in a subsidization of the primary market, sometimes resulting in prices below marginal cost. While aftermarket add-ons are now common in intellectual property products such as software, the firms usually can't charge a primary market price below zero. This paper shows that with the zero price constraint in effect, firms give more of the overall product away for free and pricing in the aftermarket is determined by the number of competitors in the primary market; despite assuming all consumers are initially unaware of the aftermarket's existence. With the constraint in effect, consumers are better off and firms can potentially earn less profit.
\end{abstract}

The author thanks Sherzod Akhundjanov and the two anonymous referees for feedback on the paper.

Citation: Ben O Smith, (2016) "Giving away the store: How the zero price constraint results in fewer add-on features", Economics Bulletin, Volume 36, Issue 2, pages 983-992

Contact: Ben O Smith - bosmith@unomaha.edu.

Submitted: February 08, 2016. Published: June 11, 2016. 


\section{Introduction}

Many software developers no longer sell products via boxed software, instead they sell it in an 'app store.' App store platforms allow consumers to easily discover software for their device or computer while managing the transaction process for the developer. One of the most popular features of the app store environment is the 'in-app purchase' - allowing developers to unlock features within their application (for a fee) after the user has used the product for some period of time. Some common in-app purchases include additional features in productivity applications and automatic level advancement in games.

As of 2015, there are more than 2.5 million mobile applications in the Apple App Store and Google Play Store alone (Martin et al., 2015). This level of competition has resulted in very low base good prices (the primary market), in comparison to software prices of the past. Official Apple numbers from 2011 suggest that $37 \%$ of all app store applications were priced at $\$ 0.00$ and the average price was $\$ 3.64$ (Elmer-DeWitt, 2011). An updated analysis in 2016 suggest that $76 \%$ of applications are free and the average price is $\$ 1.13$ (Poc, 2016).

For concreteness, consider 'To-Do' applications as an example. There are more than fifty 'To-Do' apps on the Mac App Store, most of which are free and rely heavily on in-app purchases for revenue. For instance, the most popular application is Wunderlist, which is available for free, but the user gains a slight increase in flexibility in managing very expansive to-dos and cosmetic changes after an in-app purchase. A similar story can be told with many other types of applications including social media utilities, weather dashboards, and basic photo applications.

A large number of free products in app stores is not entirely unexpected. As established in the literature, if boundedly rational consumers choose a good based on its primary market price, the firms subsidize the primary market in hopes the consumer will buy the aftermarket add-on - in this case the in-app purchase (for a literature review see Ellison (2005) and Coppi (2007)). However, what current models do not explain is the small increase in features the consumer gains by buying the add-on product (when there are many products in the primary market).

Current literature suggests that less market power in the primary market results in more of a subsidy (Borenstein et al., 2000; Gabaix and Laibson, 2006). But it is logistically impossible to charge a primary market price below zero. Therefore, there exists a 'zero price constraint' where the firm can't charge a lower price in response to increased competition. What is the firm to do facing this environment? In this paper, I will show that they will 'compete' by reducing the number of features that they migrate to the add-on product. This results in a decrease in the aftermarket price with more primary market competition, despite assuming that consumers are initially unaware of the aftermarket. In the end, fewer consumers will purchase the add-on product, some consumers will be better off and firms may experience a reduction in profit as a result of the constraint. 


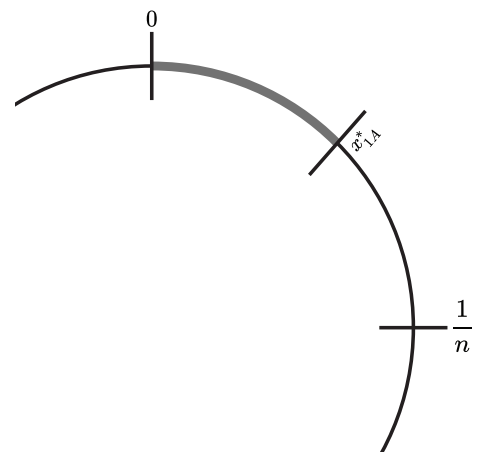

Figure 1: The indifferent consumer $\left(x_{1 A}^{*}\right)$ is found by setting the utility of buying product $A$ from Firm 1 equal to the utility of buying product $A$ from Firm $1 / n$. Any consumer between zero and $x_{1 A}^{*}$ will purchase the good from Firm 1 as the utility is greater. Therefore, demand is twice the dark grey line. The primary market and aftermarket are assumed to be independent, thus any point on the Salop circle produces a full spectrum of aftermarket positions.

\section{Model}

Assume that the consumers are boundedly rational such that they consider the expected net utility of the primary product (product $A$ ) but they don't obtain information about the aftermarket (product $B$ ). Similar to the argument by Ellison (2005, p. 609), it is infeasible for firms to advertise their competitors' add-on prices and, while customers may know that there are add-ons, the search costs are high enough that it is not worth the effort to obtain accurate aftermarket price/feature information ${ }^{1}$. Consumers are uniformly distributed in preferences around a Salop circle (1979) with circumference one. The net utility of product $A$ is assumed to be so high that outside goods are a non-issue.

As this paper's focus is on intellectual property products, one cannot assume the size of the add-on is endowed by the nature of the product (which is the more common assumption in the literature). Therefore, in this model, firms choose the number of features (utility) to transfer from product $A$ to $B$. In the aftermarket, consumers choose whether to buy $B$ or avoid the add-on. While mathematically similar to a monopoly producer, it is a refined interpretation. Assume the consumers' primary market position is independent of their position in the aftermarket.

We'll first examine the model when the primary market price is above zero and then compare it to when the 'zero price constraint' becomes binding.

\subsection{Non-Zero Primary Market Pricing}

Demand is found by locating the consumer who is indifferent between the goods of the two neighboring firms. $U_{1 A}$ is the utility of purchasing from the firm located at position zero (Firm 1) and $U_{\frac{1}{n} A}$ represents the utility of purchasing from the firm located at $1 / n$

\footnotetext{
${ }^{1}$ Oftentimes, determining add-on prices for the consumer is not the largest hurdle. Unlike durable goods, the size and scope of add-ons are artificially determined in software and the definitions are product dependent (i.e. the price of the "Pro-Pack" provides no additional information as the user doesn't know what the "Pro-Pack" includes or how they would use it). To determine the benefit of the add-on, the consumer must first become familiar with the software, often entering a large amount of user data. This creates a switching cost. In this model, I assume that the switching cost is high enough that the user does not search for another primary market product. In productivity software, this is likely the case. But a less data or experience-driven application may violate this assumption.
} 
(Firm $1 / n)$ - where $n$ is the number of firms in the industry. Setting $U_{1 A}$ equal to $U_{\frac{1}{n} A}$, we solve for the indifferent consumer (Figure 1).

$$
\begin{aligned}
\underbrace{\left(s_{A}-s_{1 B}\right)-p_{1 A}-t_{A} x_{A}}_{U_{1 A}} & =\underbrace{\left(s_{A}-s_{\frac{1}{n} B}\right)-t_{A}\left(\frac{1}{n}-x_{A}\right)-p_{\frac{1}{n} A}}_{U_{\frac{1}{n} A}} \\
x_{A}^{*} & =\frac{n\left(s_{\frac{1}{n} B}-s_{1 B}-p_{1 A}+p_{\frac{1}{n} A}\right)+t_{A}}{2 n t_{A}}
\end{aligned}
$$

where $s_{A}$ is the gross benefit to the consumer, $t_{A}$ is the travel cost and $x_{A}$ is the position of the consumer. $s_{1 B}$ represents the utility (features) transferred to the aftermarket by Firm 1 while $p_{1 A}$ represents Firm 1's price in the primary market. $s_{\frac{1}{n} B}$ and $p_{\frac{1}{n} A}$ have similar definitions for Firm $1 / n$.

While every consumer will purchase product $A$ from someone, this is not true of product $B$. $B$ is a set of features equal to utility $s_{i B}$ (for all consumers) packaged as an addon. Having purchased a product around the Salop circle, the consumer must decide if the additional utility can be obtained with an alternative solution. The consumers can expend a combination of effort and money to avoid the add-on (the alternative solution increases in quality with a higher price), but the degree to which this will satisfy the consumer will vary based on the customer's preferences/creativity - not the least of which is the degree to which they are annoyed that it doesn't integrate into the primary product. In describing how informed consumers avoid aftermarket rents, Gabaix and Laibson (2006, p. 515) made a similar argument but they didn't allow for varying abilities/preferences.

To add some concreteness to our discussion of the alternative to product $B$, consider the to-do application in the introduction (Wunderlist). The aftermarket add-on allows for an increase in the number of to-do subitems. As an alternative, the consumer could manage to-do subitems in a spreadsheet program and attach the files to each main to-do item in the application. While the utility from product $B$ is the same for all users, some technically inclined consumers who place a low value on their time might choose the alternative solution. However, some consumers might view the alternative as more costly due to a lower level of technical knowledge or a higher valuation of their time. If the price of the add-on increased substantially, all consumers would be willing to consider more elaborate or more expensive solutions; perhaps buying third party products to fulfill the same function.

To find the indifferent consumer in the aftermarket, set the utility of product $B\left(U_{1 B}\right)$ equal to the alternative $\left(U_{E}\right)$, where quality of the alternative solution is equal to the price of product $B\left(p_{i B}\right)$ and consumers with differing preferences represented by their position between zero and one $\left(x_{i B}\right)$ in a Hotelling city $(1929)^{2}$.

\footnotetext{
${ }^{2}$ While there are alternative methods of modeling this relationship, they are mathematically more complex and do not result in a different interpretation. For instance, using a multiplier on $U_{E}$ would model the idea that the alternative solution is less desirable to all consumers due to the lack of integration into the primary market good; we've achieved the same interpretation by setting the mean
} 


$$
\begin{aligned}
& \underbrace{s_{1 B}}_{U_{1 B}}=\underbrace{p_{1 B}-t_{B}\left(\frac{3}{2}-x_{1 B}\right)}_{U_{E}} \\
& x_{1 B}^{*}=\frac{3}{2}-\frac{p_{1 B}}{t_{B}}+\frac{s_{1 B}}{t_{B}}
\end{aligned}
$$

where $x_{1 B}^{*}$ describes the proportion of Firm 1's product $A$ customers that buy product $B$.

For simplicity, assume that the firms have zero marginal costs and incur development cost $f$. This is nearly the case for intellectual property products with digital distribution.

$$
\pi_{1}=2(\underbrace{\frac{n\left(s_{\frac{1}{n} B}-s_{1 B}-p_{1 A}+p_{\frac{1}{n} A}\right)+t_{A}}{2 n t_{A}}}_{x_{A}^{*}})\left(p_{1 A}+(\underbrace{\frac{3}{2}-\frac{p_{1 B}}{t_{B}}+\frac{s_{1 B}}{t_{B}}}_{x_{1 B}^{*}}) p_{1 B}\right)-f
$$

Firm 1's profit equation is the proportion of the Salop circle captured by Firm $1\left(2 x_{A}^{*}\right)$ multiplied by the product $A$ price $\left(p_{1 A}\right)$ plus the revenue from product $B . x_{A}^{*}$ is doubled due to the firms located on Firm 1's left and right on the Salop circle. The revenue from product $B$ is the proportion of the firm's product $A$ customers who buy product $B\left(x_{1 B}^{*}\right)$ multiplied by the number of product $A$ customers $\left(2 x_{A}^{*}\right)$ times price $\left(p_{1 B}\right)$.

Using a solution approach akin to that of Salop's original paper (1979), we take derivatives with respect to $p_{1 A}, p_{1 B}$ and $s_{1 B}$ (and set them equal to zero). However, as we know that all firms react identically, we can then replace the neighbor variables $p_{\frac{1}{n} A}, p_{\frac{1}{n} B}$ and $s_{\frac{1}{n} B}$ with their Firm 1 counterparts. This reveals our system of equations to find the optimal solution.

$$
\begin{aligned}
\frac{1}{n}-\frac{p_{1 A}}{t_{A}}+\frac{p_{1 B}^{2}}{t_{A} t_{B}}-\frac{p_{1 B} s_{1 B}}{t_{A} t_{B}}-\frac{3 p_{1 B}}{2 t_{A}} & =0 \\
\frac{s_{1 B}}{n t_{B}}+\frac{3}{2 n}-\frac{2 p_{1 B}}{n t_{B}} & =0 \\
\frac{p_{1 B}}{n t_{B}}-\frac{p_{1 A}}{t_{A}}+\frac{p_{1 B}^{2}}{t_{A} t_{B}}-\frac{p_{1 B} s_{1 B}}{t_{A} t_{B}}-\frac{3 p_{1 B}}{2 t_{A}} & =0
\end{aligned}
$$

This system of equations can be solved for $p_{1 A}, p_{1 B}$ and $s_{1 B}$. As all firms are identical, the "1" in the subscripts of the final solution are replaced with $i$.

of the alternative solution to $p_{i B}-t_{B}$. Similarly, one could argue that there is a portion of aftermarket utility that is fixed and not chosen by the firm $\left(s_{f}\right)$. This would result in $U_{i B}=s_{i B}+s_{f}$. Such an alteration would result in slight variations in the solutions for $s_{i B}\left(\frac{t_{B}}{2}-s_{f}\right.$ and $\frac{2 t_{A}}{n}-\frac{3 t_{B}}{2}-s_{f}$ for the unconstrained and constrained result, respectfully), but would otherwise not alter the interpretation of the model. However, when there is only a fixed utility component to the aftermarket good the solution is substantially altered. Such a model is included in the appendix. 


$$
\begin{aligned}
p_{i A} & =\frac{t_{A}}{n}-t_{B} \\
p_{i B} & =t_{B} \\
s_{i B} & =\frac{t_{B}}{2}
\end{aligned}
$$

The firm subsidizes the aftermarket by charging a lower price in the primary market and transfers enough features $\left(s_{i B}\right)$ to fully capture the aftermarket; substituting the final solutions in $5, x_{i B}^{*}=1$. In aggregate, this does not change net profit from a standard Salop circle $\left(\pi_{i}=\frac{t_{A}}{n^{2}}-f\right)$. This is similar to previous literature (e.g. Lal and Matutes (1994)) where the addition of the aftermarket has no net effect on profit. However, we should consider the limitations of location models. If the average $p_{i A}$ in the industry drops, the size of the industry likely increases, which is not reflected in the model.

\subsection{Zero Primary Market Pricing}

If $t_{B}>\frac{t_{A}}{n}$ then the price of $A$ in 5 is logistically impossible and the zero price constraint becomes binding. Assuming the primary market price is binding at zero, equation 1 can be restated as follows:

$$
\begin{aligned}
\underbrace{\left(s_{A}-s_{1 B}\right)-t_{A} x_{A}}_{U_{1 A}} & =\underbrace{\left(s_{A}-s_{\frac{1}{n} B}\right)-t_{A}\left(\frac{1}{n}-x_{A}\right)}_{U_{\frac{1}{n} A}} \\
x_{A}^{*} & =\frac{n\left(s_{\frac{1}{n} B}-s_{1 B}\right)+t_{A}}{2 n t_{A}}
\end{aligned}
$$

$s_{i B}$ is the only choice for the firm in the primary market. Restating the profit equation, the firm only earns revenue from product $B$.

$$
\pi_{1}=2(\underbrace{\frac{n\left(s_{\frac{1}{n} B}-s_{1 B}\right)+t_{A}}{2 n t_{A}}}_{x_{A}^{*}})(\underbrace{\frac{3}{2}-\frac{p_{1 B}}{t_{B}}+\frac{s_{1 B}}{t_{B}}}_{x_{1 B}^{*}}) p_{1 B}-f
$$

Taking derivatives with respect to $p_{1 B}$ and $s_{1 B}$ and replacing neighbor variables reveals the system of equations.

$$
\begin{aligned}
\frac{s_{1 B}}{n t_{B}}+\frac{3}{2 n}-\frac{2 p_{1 B}}{n t_{B}} & =0 \\
\frac{p_{1 B}}{n t_{B}}+\frac{p_{1 B}^{2}}{t_{A} t_{B}}-\frac{p_{1 B} s_{1 B}}{t_{A} t_{B}}-\frac{3 p_{1 B}}{2 t_{A}} & =0
\end{aligned}
$$




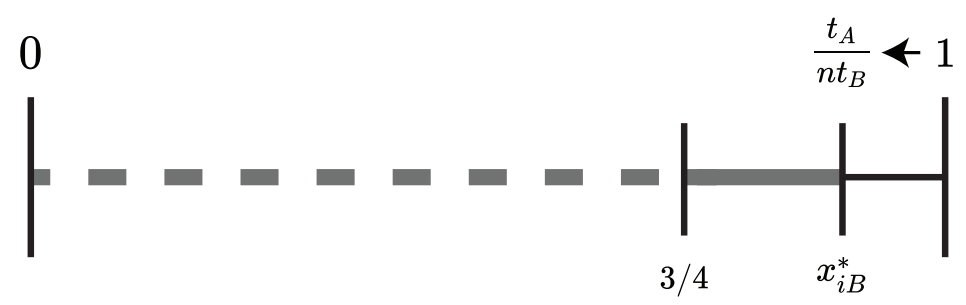

Figure 2: The aftermarket is a choice between buying the add-on and the alternative (avoidance). Utility from avoidance varies from consumer to consumer. Finding the indifferent consumer finds the proportion of consumers willing to buy the add-on. The indifferent consumer is located at 1 when the zero price constraint is unbinding. With a binding zero price constraint, the indifferent consumer is located at $\frac{t_{A}}{n t_{B}}$. The dark gray line represents the proportion of product $A$ customers who buy product $B$.

With two equations in 8 , the system can be solved for the two unknowns $p_{1 B}$ and $s_{1 B}$. As in the the non-zero pricing section, I've replaced the " 1 " with an $i$ in the final solution.

$$
\begin{aligned}
& p_{i B}=\frac{t_{A}}{n} \\
& s_{i B}=\frac{2 t_{A}}{n}-\frac{3 t_{B}}{2}
\end{aligned}
$$

Unlike the unconstrained problem, pricing in the aftermarket depends on $n$. Further, the aftermarket is not fully captured; substituting the final solutions in $9, x_{i B}^{*}=\frac{t_{A}}{n t_{B}}$, which is less than one when the zero price constraint is binding $\left(t_{B}>\frac{t_{A}}{n}\right)$ and decreases with more competition $^{3}$ (Figure 2). Similarly, rearranging $t_{B}>\frac{t_{A}}{n}$ in 10 shows that fewer features are transferred to the aftermarket.

$$
t_{B}>\frac{t_{A}}{n} \Rightarrow 0>\frac{2 t_{A}}{n}-2 t_{B} \Rightarrow \underbrace{\frac{t_{B}}{2}}_{\begin{array}{c}
s_{i B} \text { with non-zero } \\
\text { primary market price }
\end{array}}>\underbrace{\frac{2 t_{A}}{n}-\frac{3 t_{B}}{2}}_{\begin{array}{c}
s_{i B} \text { with zero } \\
\text { primary market price }
\end{array}}
$$

This decrease in aftermarket features results in a decrease in aftermarket sales. The relationship between aftermarket features and add-on sales can be explored by taking the difference in $s_{i B}$ in the absence of a zero price constraint and when the constraint is binding:

$$
\Delta s_{i B}=\underbrace{\frac{t_{B}}{2}}_{\begin{array}{c}
s_{i B} \text { with non-zero } \\
\text { primary market price }
\end{array}}-\underbrace{\left(\frac{2 t_{A}}{n}-\frac{3 t_{B}}{2}\right)}_{\begin{array}{c}
s_{i B} \text { with zero } \\
\text { primary market price }
\end{array}} \Rightarrow \Delta s_{i B}=2 t_{B}(1-\underbrace{\frac{t_{A}}{n t_{B}}}_{x_{i B}^{*}})
$$

As characterized by equation 11, fewer aftermarket features results in fewer aftermarket sales (shown by a decrease in $\frac{t_{A}}{n t_{B}}$ with an increase in $\Delta s_{i B}$ ). This deviation from the

\footnotetext{
${ }^{3} x_{B}^{*}$ is bounded between $3 / 4$ and 1. $s_{i B}$ is negative if $\frac{t_{A}}{n t_{B}}<3 / 4$.
} 
unconstrained solution is also reflected in the final profit equation:

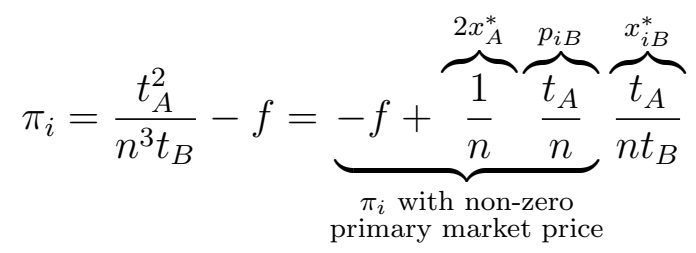

When $x_{i B}<1$ the firm earns less profit than when the zero price constraint is unbinding. Given $t_{B}>\frac{t_{A}}{n}$ when the zero price constraint is binding, this is always true.

\section{Discussion}

Due to the fixed industry size assumption, a formal surplus analysis can be problematic with location models. However, the prices of the two products reveal a surprising amount of information. All consumers purchase both the primary market product and aftermarket good for a total of $\frac{t_{A}}{n}$ when the zero price constraint is unbinding. For the proportion of consumers who buy the aftermarket good when the zero price constraint is in effect $\left(\frac{t_{A}}{n t_{B}}\right)$, there is no net change in welfare as the total price and utility remain the same. However, those who do not buy the good are made better off as they reap the benefits of the primary market good and choose to avoid the add-on (which, for them, is optimal).

While the average consumer is better off when the zero price constraint is in effect, firm profit isn't as optimistic. Assuming the industry size is fixed, firms experience a profit reduction as a function of reduced aftermarket sales. Under this fixed industry size assumption, the firms are worse off in comparison to both when the zero price constraint isn't binding and when there is no aftermarket (which comes to the same net price and profit as the unconstrained model). A simple algebraic rearrangement shows that for the firms in a zero price constraint environment to be as well off as they would have been without an aftermarket, the industry size must increase by a factor of $\frac{n t_{B}}{t_{A}}$. While such growth may occur as a result of the decrease in the industry average primary market price from $\frac{t_{A}}{n}$ to zero, it is not guaranteed.

\section{Conclusion}

The zero price constraint has four important effects: (1) fewer features are transferred to the aftermarket (2) fewer consumers buy the add-on (3) the add-on price decreases as a function of primary market competition and (4) profit is lower under some conditions. Without the ability to the lower the primary market price, the firms resort to increasing the features of the free product, which reduces the utility of the add-on. With the lower utility obtained from the aftermarket product, fewer customers can justify the purchase, particularly when the primary market is more competitive. This leads to lower profit for the firm when the industry is of a fixed size. In fact, while some product categories may 
benefit from in-app purchases, there may be some categories that are worse off. As the presence of app stores expands from mobile devices to other environments such as desktop and television (Fowler, 2015; Kane and Sherr, 2011; Pogue, 2012), one must consider the implications of the zero price constraint.

\section{References}

(2016) "App Store Metrics." Pocket Gamer, Available: http://goo.gl/hGysl1 [Online; updated April-2016].

Borenstein, S., MacKie-Mason, J.K. and Netz, J.S. (2000) "Exercising Market Power In Proprietary Aftermarkets." Journal of Economics 63 Management Strategy 9, 157-188.

Coppi, L. (2007) "Aftermarket Monopolization: The Emerging Consensus In Economics." The Antitrust Bulletin 52, 53-71.

Ellison, G. (2005) "A Model of Add-On Pricing." The Quarterly Journal of Economics 120, 585-637.

Elmer-DeWitt, P. (2011) “Apple's iTunes store: 500,000 iOS apps and counting." Fortune, Available: http://for.tn/1goa0yc [Online; posted 24-May-2011].

Fowler, G. (2015) "Apple TV Review: A Giant iPhone for Your Living Room." The Wall Street Journal, Available: http://on.wsj.com/1GxR79D [Online; posted 28-October2015].

Gabaix, X. and Laibson, D. (2006) "Shrouded Attributes, Consumer Myopia, And Information Suppression In Competitive Markets." The Quarterly Journal of Economics 121, 505-540.

Hotelling, H. (1929) "Stability in Competition." The Economic Journal 39, 41-57.

Kane, Y. and Sherr, I. (2011) "Apple Takes Wraps Off Mac App Store." The Wall Street Journal, Available: http://on.wsj.com/1zFywjn [Online; posted 07-January-2011].

Lal, R. and Matutes, C. (1994) "Retail Pricing And Advertising Strategies." Journal of Business 67, 345-370.

Martin, W., Harman, M., Jia, Y., Sarro, F. and Zhang, Y. (2015) "The App Sampling Problem for App Store Mining." In 12th Working Conference on Mining Software Repositories (MSR).

Pogue, D. (2012) "A Review of the Windows 8 Beta." The New York Times, Available: http://nyti.ms/NXnceb [Online; posted 29-February-2012].

Salop, S. (1979) "Strategic Entry Deterrence." The American Economic Review 69, $335-338$. 


\section{Appendix: Fixed Utility of the Aftermarket Good}

An astute referee suggested a variation on the model where the aftermarket product could not be provided as part of the primary market product. Instead the aftermarket good's utility is fixed (denoted as $s_{f}$ in this model). The best example of such a product would be a video game where a frustrated customer is offered the opportunity to skip a level if they pay a fee. Such a product would be nearly costless to provide, but would be only appealing to customers who have already purchased the primary market product.

This variation to the model modifies the primary market indifferent consumer to the following:

$$
x_{A}^{*}=\frac{n\left(p_{\frac{1}{n} A}-p_{1 A}\right)+t_{A}}{2 n t_{A}}
$$

With this modified indifferent consumer the new profit equation for Firm 1 would be:

$$
\pi_{1}=2\left(\frac{n\left(p_{\frac{1}{n} A}-p_{1 A}\right)+t_{A}}{2 n t_{A}}\right)\left(p_{1 A}+\left(\frac{3}{2}-\frac{p_{1 B}}{t_{B}}+\frac{s_{f}}{t_{B}}\right)\right)-f
$$

For brevity, I will provide the final solutions and profit:

$$
\begin{aligned}
p_{i A} & =\frac{t_{A}}{n}-\underbrace{\frac{\left(2 s_{f}+3 t_{B}\right)^{2}}{16 t_{B}}}_{\Lambda} \\
p_{i B} & =\frac{s_{f}}{2}+\frac{3 t_{B}}{4} \\
\pi_{i} & =\frac{t_{A}}{n^{2}}-f
\end{aligned}
$$

When the zero price constraint is binding, the profit equation is simplified further:

$$
\pi_{1}=\frac{1}{n}\left(\frac{3}{2}-\frac{p_{1 B}}{t_{B}}+\frac{s_{f}}{t_{B}}\right) p_{1 B}-f
$$

This results in the following price and profit solution:

$$
\begin{aligned}
p_{i B} & =\frac{s_{f}}{2}+\frac{3 t_{B}}{4} \\
\pi_{i} & =\frac{1}{n} \underbrace{\frac{\left(2 s_{f}+3 t_{B}\right)^{2}}{16 t_{B}}}_{\Lambda}-f
\end{aligned}
$$

Notably the two $\Lambda$ sections are identical. Therefore, we can compare the two profits in the following way: 


$$
\frac{t_{A}}{n^{2}}-f \stackrel{?}{<} \frac{1}{n} \Lambda-f \Rightarrow \frac{t_{A}}{n^{2}} \stackrel{?}{<}>\frac{1}{n} \Lambda \Rightarrow \frac{t_{A}}{n} \stackrel{?}{<}>\Lambda
$$

Therefore, when $p_{i A}$ is negative $\left(\frac{t_{A}}{n}<\Lambda\right)$ and the zero price constraint is binding, the constrained solution is more profitable. Otherwise, the unconstrained solution is more profitable. 